\title{
A Payer Perspective of the Hospital Inpatient Additional Care Costs of Antimicrobial Resistance in France: A Matched Case-Control Study
}

\author{
Mehdi Touat $^{1} \cdot$ Marion Opatowski $^{1} \cdot$ Christian Brun-Buisson $^{1} \cdot$ Kristel Cosker $^{2} \cdot$ Didier Guillemot $^{1}$ - Jerome Salomon ${ }^{3}$. \\ Philippe Tuppin $^{4} \cdot$ Gregoire de Lagasnerie $^{5} \cdot$ Laurence Watier $^{1}$ (1)
}

Published online: 3 December 2018

(c) The Author(s) 2018

\begin{abstract}
Background and Objective Antimicrobial resistance (AMR) has become one of the biggest threats to global public health given its association with mortality, morbidity and cost of health care. However, little is known on the economic burden of hospitalization attributable to AMR from a public health insurance perspective. We assessed the excess costs to the French public health insurance system attributable to AMR infections in hospitals.

Methods Bacterial infectious disease-related hospitalizations were extracted from the National health data information system for all stays occurring in 2015. Bacterial infections, strains, and microbial resistance were identified by specific French ICD-10 codes. Information about health care expenditure, co-morbidities and demographic characteristics (i.e. gender, age) are provided. We used a matched case-control approach to determine the excess of reimbursements paid to stays with AMR compared to stays with an infection without resistance. Cases and controls were matched on gender, age, Charlson comorbidity index, category of infection, infection as principal diagnosis (two classes), microorganism and hospital status. The overall AMR cost was extrapolated to stays with AMR and excluded from the sample (multiple infections), and a second extrapolation was performed to consider stays with unknown resistance status.

Results The final sample included 52,921 matched-pairs (98.2\% cases). Our results suggest that AMR overall cost reached EUR109.3 million in France with a mean of EUR1103 per stay; extrapolation to the entire database shows that the overall cost could potentially reach EUR287.1 million if all cases would be identified. The mean excess length of hospital stay attributable to AMR was estimated at 1.6 days.

Conclusion AMR causes substantial cost burden in France for the public health insurance. Our study confirms the need to reinforce programs to prevent AMR infection and thereby reduce their economic burden.
\end{abstract}

Electronic supplementary material The online version of this article (https://doi.org/10.1007/s40258-018-0451-1) contains supplementary material, which is available to authorized users.

Laurence Watier

laurence.watier@inserm.fr

1 Biostatistics, Biomathematics, Pharmacoepidemiology and Infectious Diseases (B2PHI), Inserm, UVSQ, Institut Pasteur, Paris-Saclay University, 2, avenue de la Source de la Bièvre, 78180 Montigny-Le-Bretonneux, France

2 Department of Biostatistics, Public Health Department, Medical Information, AP-HP University Hospitals Pitié Salpêtrière-Charles Foix, 47-83 Boulevard de l'Hôpital, 75013 Paris, France
3 Ministry of Social Affairs and Health, 14 Avenue Duquesne, 75350 Paris, France

4 Department of Studies on Patients and Diseases, CNAM (National Health Insurance), 50 Avenue du Professeur André Lemierre, 75986 Paris Cedex 20, France

5 Social Security Directorate, Ministry of Social Affairs and Health, 14 Avenue Duquesne, 75350 Paris, France 


\section{Key Points for Decision Makers}

Antimicrobial resistance (AMR) is associated with higher mortality, morbidity, longer hospital stays and higher medical resource consumption. Little is known on the burden of AMR from a public health insurance perspective on a national level.

This investigation highlights the hospital costs of antibiotic resistance derived from a medico-administrative database. AMR costs were stratified per anatomical site and microorganism. These data provide a complete economic description of the hospital costs attributable to AMR from a payer perspective.

The economic weight of AMR, corresponding to $1,648,566$ stays with infectious disease identified in the database, may amount up to $3.2 \%$ of total health expenditures. Such evaluation should inform discussions on the cost-benefit ratio of public health policies and incentivize decision makers to focus on this particular population with AMR infection to promote measures to prevent and control AMR.

\section{Introduction}

Antimicrobial resistance (AMR) is associated with more difficult-to-treat infections and potentially jeopardizes medical progress and innovation [1,2]. AMR is associated with higher mortality [3], longer hospital stays, and higher medical resource consumption [4]. A technical report from the European Commission claimed that mortality attributed to AMR reached 25,000 deaths in 2007 in the European Union [5]. In France, this mortality was evaluated by the French Public Health Agency (Santé Publique France) at 12,500 deaths in 2012 (with $7.9 \%$ infections due to multidrugresistant bacteria) [6]. Increasingly high numbers of infections with antibiotic-resistant strains have been recorded in Europe [7, 8] and in the USA [9, 10]. This increasing prevalence, the excess of mortality and extra cost caused by infections due to resistant bacteria are likely to have a major impact on national health systems. Thus, AMR is considered to be a major threat to public health [2].

Economic evaluations help to prioritize health care policies regarding AMR $[2,11]$ and to promote the prevention of these infections. However, little is known about the specific cost of AMR on the national level [12]. Hospital-based single-center studies $[4,13-16]$ were mainly used to provide a wide range of costs from the hospital perspective. The disparity in results could be related to the perspective of the study, adjustment of confounding factors, choice of the infectious site, the bacteria of interest, or the scope of hospital costs being taken into account $[12,17]$. For example, excess cost estimate for infection due to extendedspectrum $\beta$-lactamase (ESBL)-producing bacteria reached - US\$2081 [-EUR1905 (conversion to 2015 euros)] to US\$10,154 (EUR9295) per case [18]. Several European studies used a payer's perspective based on DRG-payment system (diagnosis-related group) to calculate the additional costs of AMR in hospitals [19, 20]. From a Norwegian surveillance system and a patient registry, hospital costs for inpatients with methicillin-resistant Staphylococcus aureus (MRSA) reached EUR13,233, while in the control group not diagnosed with MRSA, the hospital cost estimated through the DRG-cost was EUR 7198 [19]. In a Swiss 2200-bed hospital, the average bed-day cost for MRSA-infected patients was estimated to be 1.5 -fold higher when compared with the general population hospitalized in acute wards [20]. In a German study, focusing on MRSA whatever the anatomical location of infections, an extra length of stay of 11 days and an additional cost of EUR8198 was found [21]. Therefore, there is a lack of empirical data which would include all germs and all anatomical locations of infection.

In France, the National Hospital Discharge database (PMSI) provides comprehensive medical information about inpatients. Discharge summaries of hospital stays from PMSI are used for the funding of all hospitals by a DRG-based prospective payment [22]. It reflects the exact health expenditure from the French public health insurance perspective. Since 2015, specific codes for antimicrobial resistance markers are available in the PMSI through the International Classification of Diseases, 10th French revision (ICD-10th), which may allow estimating the hospital costs of antibiotic resistance from the payer perspective.

Co-morbidities increase both the risk of infection with antibiotic-resistant bacteria and the hospital costs of care. Therefore, an analysis that does not take them into account could overestimate the cost directly attributable to AMR. In that context, a matched case-control study design, an approach which has been widely used in this area [14, 23, 24], appears most appropriate to estimate the specific cost of AMR.

The objective of this study was to estimate the hospital cost attributable to AMR in 2015, from a health insurance perspective, using nationwide population data and a matched case-control study.

\section{Material and Methods}

\subsection{Data Sources}

The PMSI database contains all discharge summaries of hospitalization and covers all hospital stays in publicly 
funded and private (i.e. for profit) hospitals in France [25]. For acute-care facilities (medicine, surgery and obstetrics: MSO), available information includes patient characteristics (gender and age), primary, related and associated diagnoses using ICD-10 codes, length of stay, medical procedures, intensive care units (ICU) stay, and, healthcare expenditure based on DRG [25] and specific costs related to a list of innovative drugs and medical devices. Care provided by the private sector is reimbursed by the public health insurance on the basis of the same DRG-based system as the public sector. Diagnoses are coded in three categories, as: (1) the principal diagnosis (PD), which is the main reason for hospitalization; (2) related diagnoses (RD), which describe the medical context, especially when the main diagnosis is not a disease; and (3) significant associated diagnoses (SAD), which include all comorbidities influencing patients' care. Antibiotic resistance markers are coded in the ICD-10th French revision according to the WHO (World Health Organization) and the French Agency for information on hospital care (ATIH) nomenclature.

\subsection{Study Population}

The study population included all hospitalizations with an acute infection caused by Staphylococcus, Enterobacteriaceae, Streptococcus and other Gram-negative bacteria identified in 2015 from the PMSI database. For that purpose, more than 800 ICD-10 codes were retained in collaboration with clinicians and PMSI specialists. Acute infections were classified by clinicians into 13 infection sites: (1) urinary and genital tract, (2) devices and prosthesis-related infection, (3) skin and soft tissues, (4) lower respiratory tract, (5) bacteremia and sepsis (alone), (6) gastrointestinal and abdominal, (7) bone and joint, (8) during pregnancy, (9) heart and mediastinum, (10) infection in newborn, (11) ear, nose and throat, (12) eye, and (13) nervous system. In the same way, pathogens involved were classified into 9 classes: (1) Escherichia coli, (2) Klebsiella, (3) other Enterobacteriaceae, (4) S. aureus, (5) others Staphylococcus, (6) Pneumococcus, (7) Enterococcus, (8) other Streptococcus, (9) Gram-negative bacilli (GNB).

All hospital stays from 2015 with a code of interest in PD, $\mathrm{RD}$ or SAD were extracted from the anonymized individual PMSI database that also records individual demographic information, healthcare and drug reimbursements. Therefore, gender, age, length of stay, diagnosis, cost, in-hospital death and ICU stay were also collected from the database. Only stays ( $>1$ day) with an admission between January 1 and December 31, 2015 were retained.

Stays with at least one infection code were first selected $(N=1,648,566)$ (Fig. 1). Of these stays, only those for which the responsible agent could be identified were retained for the case-control analysis $(N=599,225)$. They were divided into two groups: no resistance code $(N=493,714)$ versus at least one resistance code $(N=105,511)$. Stays with a resistance code were defined as cases and those without a resistance code as controls. If several resistance codes existed, the resistance category was considered as 'unknown'. Finally, except for a few specific codes, the infection, their responsible agent and the presence of bacterial resistance cannot be linked within individual records. In this context, the sample was restricted to stays with a single infection and a single pathogen $(N=372,148)$, resulting in a final sample of 318,234 controls and 53,914 cases.

\subsection{Cost of Antimicrobial Resistance Estimations}

A public health insurance perspective was chosen for the analysis. Costs were estimated using official DRG, which includes the following expense items: medical procedures, nursing care, administration, routine drug consumption, and room service. Cost from expenses of innovative drugs for the National Health Insurance Funds and expenditure from transfer in ICU were added to DRG. Costs were estimated for both cases and controls until discharge.

Attributable cost of expenditure related to antimicrobial resistance was estimated as the additional costs of cases compared to controls, using a matched case-control approach, from the difference in costs for each matched pair. To avoid cost misallocation and identify clinically comparable groups with regard to co-morbidities and underlying severity of illness diseases, the following 7 matching criteria were used in order: gender, age ( 8 classes: $<7$ days; [8 days-28 days]; [29 days-1 year]; [2-25]; [26-35]; [36-45]; [46-55]; [56-65]; [66-75]; [75-95]; + 95 years), the Charlson index for comorbidities [4 classes: 0 ; (1-2); (3-4); $\geq 5$ ] [27], site of infection (13 classes, as previously mentioned), pathogen involved (9 classes, as previously mentioned), infection ICD-10 code category ( 2 classes: one with infection coded as PD, and the other as RD or SAD) and hospital status (public or private). To match a maximum number of cases, and obtain a balanced number of pairs, one control was exactly matched to a case on these seven criteria.

Finally, a two-step cost extrapolation was carried out to estimate the cost burden of resistance at the nationwide level. A first extrapolation was performed by including cases which had been excluded from the sample with AMR because of multiple infections. The global mean cost of AMR per case was extrapolated on these stays by age and gender. A second extrapolation was performed on stays with infection and no coded microorganism. The distribution of resistance and its cost from the derivation sample was extrapolated to these stays, according to gender, age and infection site. The total cost thus obtained by adding those of the derivation sample and extrapolated samples, provides an estimate of the total cost of the AMR if all cases with 


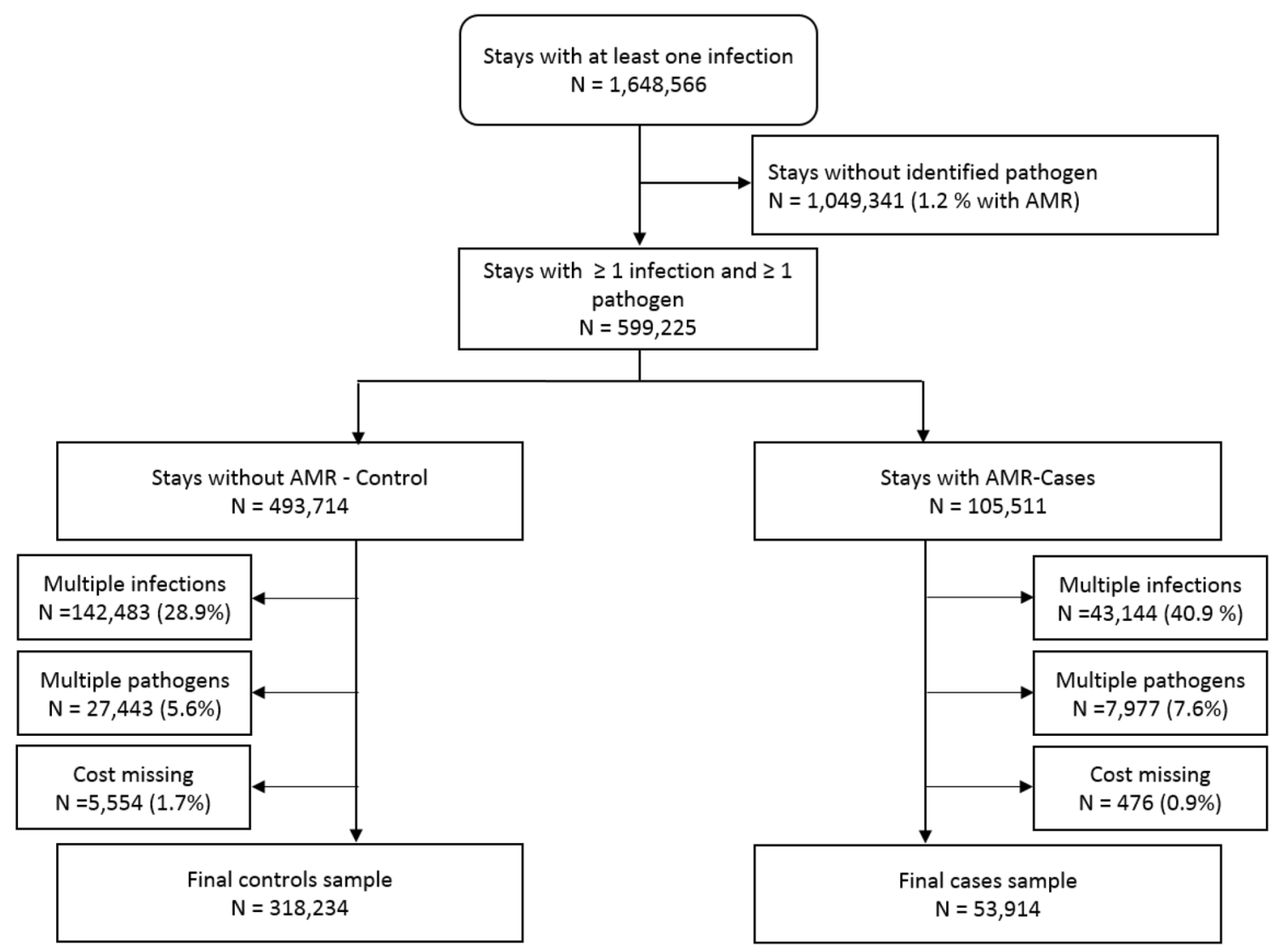

Fig. 1 Flowchart of the sample definition

infection had coding for an infection site and a microorganism identified in the PMSI.

\subsection{Analysis}

For all stays of our selected sample, we describe baseline characteristics of stays with and without AMR (gender, age, Charlson index, category of PD, and distribution of infection location). Clinical outcomes, including all-causes mortality, rate of ICU admission, length of stay, overall costs of stay. Costs (median and mean) were analyzed for matched-cases and -controls. Differential costs were estimated and stratified by infectious sites with at least 1000 stays. The $95 \%$ confidence intervals (95\% CI) using the normal distribution are given as measure of the precision of the estimates. We estimated differential costs and excess hospital stay for three main categories of pathogens and resistance (ESBL, E. coli and Klebsiella, and MRSA). For each pathogen-resistance pair, we described results for their three most frequent infection sites and for bacteremia without identified related site (i.e. primary bacteremia).

In sensitivity analyses, we also performed an unbalanced matching (1-5 controls per case) and, in order to account for unmatched cases, a multivariate linear regression (including the seven criteria used in the matching approach as cofactors). Statistical analyses were performed using SAS Enterprise Guide 9.4 (SAS Institute).

\subsection{Ethical Approval}

The study was authorized by the French data Protection agency (Commission nationale informatique et libertés [CNIL]) (REF DE-2016-176).

\section{Results}

\subsection{Baseline and Clinical Characteristics}

A sample of 372,148 acute care stays was retained in the derivation sample, including 53,914 (14.5\%) with an identified resistance marker (Fig. 1). Following the matching procedure, 52,921 pairs were available for analysis; thus, leaving only $1.8 \%$ non-matched cases (Supplementary Appendix 1) with more severe outcomes than matched cases (Supplementary Appendix 2). Within matched pairs, AMR infection was responsible for 23,302 (44.0\%) hospitalizations, i.e. with an infection as the PD. As expected, matched variables 
were quite similar between cases and controls (Table 1). For the matched pairs, the population sample had a mean $( \pm$ SD) age of $66.0( \pm 24)$ years and comprised a majority of female (53.3\%) patients. The mean Charlson index was $1.7( \pm 2.1)$. The most frequent sites of AMR infection were the urinary and gynecological tracts $(55.6 \%)$, followed by device-associated infections (12.9\%), skin and soft tissues (7.8\%), lower respiratory tract (7.3\%) infection and bacteremia and sepsis (without any other recorded site) (5.8\%). The in-hospital mortality rate reached $7.0 \%$ among stays

Table 1 Baseline characteristics of the sample in the derivation cohort

\begin{tabular}{lll}
\hline Characteristic & $\begin{array}{l}\text { Matched cases } \\
(N=52,921)\end{array}$ & $\begin{array}{l}\text { Matched } \\
\text { controls } \\
(N=52,921)\end{array}$ \\
\hline Sex, $n(\%)$ of male & $24,795(46.7)$ & $24,795(46.7)$ \\
Age, mean \pm SD in years & $66.0 \pm 24$ & $66.0 \pm 24$ \\
Charlson index, mean \pm SD & $1.7 \pm 2.1$ & $1.7 \pm 2.1$ \\
Infection as principal diagnosis, $n$ & $23,302(44.0)$ & $23,302(44.0)$ \\
$(\%)$ & & \\
Hospital status, $n(\%)$ of public & $43,411(82.0)$ & $43,411(82.0)$ \\
Location of infection, $n(\%)$ & & \\
Urinary and gynecological tract & $29,414(55.6)$ & $29,414(55.6)$ \\
Material infection & $6853(12.9)$ & $6853(12.9)$ \\
Skin and soft tissue & $4135(7.8)$ & $4135(7.8)$ \\
Lower respiratory tract & $3866(7.3)$ & $3866(7.3)$ \\
Bacteremia and sepsis (alone) & $3057(5.8)$ & $3057(5.8)$ \\
Gastrointestinal and abdominal & $2484(4.7)$ & $2484(4.7)$ \\
Bone and joint & $1327(2.5)$ & $1327(2.5)$ \\
During pregnancy (mother) & $681(1.3)$ & $681(1.3)$ \\
Infection in newborn & $423(0.8)$ & $423(0.8)$ \\
Heart and mediastinum & $412(0.8)$ & $412(0.8)$ \\
Ear, nose and throat & $159(0.3)$ & $159(0.3)$ \\
Eye & $72(0.1)$ & $72(0.1)$ \\
Nervous system & $38(0.1)$ & $38(0.1)$ \\
\hline
\end{tabular}

with AMR infection, and 5.8\% among those without AMR marker identified (Table 2). ICU stays occurred at least once for 5735 (10.8\%) stays with AMR compared with 5405 (10.2\%) without AMR. The median (first quartile-third quartile) length of stay was $9(5-16)$ days for infection with AMR and 8 (4-14) days without AMR.

\subsection{Economic Analysis}

The median hospital cost per stay of matched-cases amounted to EUR4767 (Table 2) with 25\% costing more than EUR8003; because of the skewness of cost distribution, the mean cost was higher and reached EUR7322. When the main reason for hospitalization was an infection, the median and mean costs per stay decreased to EUR3758 and EUR5070, respectively.

The mean difference of hospital costs between matchedpairs was EUR1103 in favor of matched-cases, leading to a total excess expenditure related to AMR of EUR58.3 million with an associated extra length of hospital days equal to 81,018 days. The interquartile range was EUR 4471, and $41 \%$ of matched-pairs were below EUR0. Differential costs for infection as a PD were lower than cost of stays with infection as associated diagnosis. Also, the additional cost was higher in the public sector whereas the number of hospital days in excess was the same in both sectors (Supplementary Appendix 3).

Across the most frequent infectious sites (Table 3), differential mean costs varied by a factor of 4 . Indeed, the lowest cost equaled EUR691.1 per case for urinary and gynecological site, with an excess hospital stay of 1 day, and the highest amounted to EUR2778.2 per case for primary bacteremia, with an excess hospital stay of 2.3 days. Lower respiratory tract infections were associated with the highest excess hospital stay recorded (3 days), although their associated differential cost (about EUR1800) was less than

Table 2 Clinical outcomes and hospital charges for stays in the 2 study groups

\begin{tabular}{|c|c|c|}
\hline & Matched-cases $(N=52,921)$ & Matched-controls $(N=52,921)$ \\
\hline In-hospital mortality (all causes) $n(\%)$ & $3726(7.0)$ & $3078(5.8)$ \\
\hline Transfer in ICU, $n(\%)$ & $5735(10.8)$ & $5405(10.2)$ \\
\hline Length of stay-median $[\mathrm{Q} 1-\mathrm{Q} 3]^{\mathrm{a}}$ in days & $9[5-16]$ & $8[4-14]$ \\
\hline Cost of stays $(\text { all })^{\mathrm{b}}-$ median $[\mathrm{Q} 1-\mathrm{Q} 3]$ in euros & $4767[3006-8003]$ & $4253[2577-7078]$ \\
\hline Cost of stay $(\mathrm{PD})^{\mathrm{c}}$-median $[\mathrm{Q} 1-\mathrm{Q} 3]$ & 3758 [2399-6082] & $3041[1406-4891]$ \\
\hline Cost of stay (all)-mean \pm SD & $7322 \pm 10,188$ & $6219 \pm 8141$ \\
\hline Cost of stay (PD)-mean $\pm \mathrm{SD}$ & $5120 \pm 5533$ & $3843 \pm 4583$ \\
\hline
\end{tabular}

$P D$ principal diagnosis, $I C U$ intensive care unit, $S D$ standard deviation

${ }^{\mathrm{a}} 25$ th percentile- 75 th percentile

${ }^{\mathrm{b}}$ All stays

${ }^{\mathrm{c}}$ Stays with infection as principal diagnostic 
Table 3 Average attributable costs and excess hospital days per stay, in euro (Matching 1:1)

\begin{tabular}{lrllr}
\hline Infectious sites & $N$ & $\begin{array}{l}\text { Differential cost } \\
(\text { mean 95\% CI) }\end{array}$ & $\begin{array}{l}\text { Hospital days in } \\
\text { excess (mean 95\% } \\
\text { CI) }\end{array}$ & Total expenditure \\
\hline Urinary and gynecological tract & 29,414 & $691[619-762]$ & $1.0[0.9-1.1]$ & $20,326,982$ \\
Material infection & 6853 & $1088[774-1401]$ & $2.0[1.5-2.5]$ & $7,455,404$ \\
Skin and soft tissue & 4135 & $1082[764-1399]$ & $1.9[1.4-2.4]$ & $4,474,034$ \\
Lower respiratory tract & 3866 & $1835[1216-2452]$ & $3.0[2.3-3.7]$ & $7,092,778$ \\
Bacteremia and sepsis (alone) & 3057 & $2778[1984-3572]$ & $2.3[1.4-3.2]$ & $8,492,816$ \\
Gastrointestinal and abdominal & 2484 & $1308[856-1759]$ & $1.7[1.1-2.3]$ & $3,249,079$ \\
Bone and joint & 1327 & $1937[1592-2282]$ & $1.5[0.7-2.3]$ & $2,568,590$ \\
Others & 1785 & $2626[1823-3429]$ & $3.9[2.8-5.0]$ & $4,689,619$ \\
All sites & 52,921 & $1103[1006-1198]$ & $1.5[1.4-1.6]$ & $58,349,305$ \\
\hline
\end{tabular}

CI confidence interval

${ }^{a}$ Others included: nervous system, eye, heart and mediastinum, ear, nose and throat infections, infections during pregnancy and infection in newborn the maximum. Finally, the total expenditure measured in the sample amounted to EUR58 million.

Among MRSA, ESBL E. coli or Klebsiella infections (Table 4), the highest differential cost was observed for
ESBL Klebsiella, with a mean cost of EUR1754 per case and the lowest cost was found with ESBL E. coli with EUR955. When MRSA was involved, the differential costs varied little according to the anatomical site of infection, varying from

Table 4 Differential cost and excess hospital days by three most frequent infection sites and bacteremia for the three main pairs of pathogens and resistance

\begin{tabular}{|c|c|c|c|c|}
\hline Resistant pathogens & $N$ & $\begin{array}{l}\text { Cost of stays in euro (cases) } \\
\text { (mean } 95 \% \mathrm{CI} \text { ) }\end{array}$ & $\begin{array}{l}\text { Differential cost in euro } \\
\text { (mean } 95 \% \mathrm{CI} \text { ) }\end{array}$ & $\begin{array}{l}\text { Hospital days in } \\
\text { excess (mean } 95 \% \\
\text { CI) }\end{array}$ \\
\hline MRSA (all infections) & 7879 & $9166 \pm 9556$ & 1553 [1301-1805] & $2.0[1.6-2.4]$ \\
\hline Skin and soft tissues & 2238 & $6931 \pm 7915$ & 1487 [1077-1898] & $2.1[1.4-2.8]$ \\
\hline Material infection & 1716 & $10,514 \pm 9175$ & 1547 [1007-2087] & $2.3[1.4-3.2]$ \\
\hline Urinary and gynecological tract & 1252 & $7074 \pm 7374$ & 1424 [956-1892] & $1.9[0.9-2.9]$ \\
\hline Bacteremia and sepsis (alone) & 695 & $11,368 \pm 10,448$ & $1688[660-2715]$ & $1.3[-0.2-2.9]$ \\
\hline Other sites ${ }^{\mathrm{a}}$ & 1978 & $11,077 \pm 11,485$ & 1666 [1069-2262] & $2.2[1.3-3.1]$ \\
\hline E. coli ESBL (all infections) & 17,168 & $5728 \pm 7025$ & $955[840-1070]$ & $1.2[1.0-1.4]$ \\
\hline Urinary and gynecological tract & 13,200 & $4804 \pm 4884$ & 788 [690-885] & $0.9[0.7-1.1]$ \\
\hline Gastrointestinal and abdominal & 1028 & $7304 \pm 10,112$ & 1439 [821-2058] & $1.3[0.5-2.1]$ \\
\hline Material infection & 1013 & $9001 \pm 10,206$ & $1274[585-1963]$ & $2.3[1.3-3.3]$ \\
\hline Bacteremia and sepsis (alone) & 606 & $11,149 \pm 13,143$ & 2559 [1410-3708] & $2.3[0.9-3.7]$ \\
\hline Other sites ${ }^{b}$ & 1321 & $8739 \pm 11,106$ & $1271[570-1972]$ & $2.1[1.2-3.0]$ \\
\hline Klebsiella ESBL (all infections) & 3759 & $8238 \pm 12,521$ & 1754 [1329-2180] & $2.5[1.9-3.1]$ \\
\hline Urinary and gynecological tract & 2570 & $5661 \pm 6412$ & 919 [712-1125] & $1.5[0.9-2.1]$ \\
\hline Material infection & 361 & $11,228 \pm 13,809$ & 2868 [1901-3836] & $3.6[1.3-5.9]$ \\
\hline Lower respiratory tracts & 288 & $19,291 \pm 25,986$ & $5448[3351-7545]$ & $5.1[1.9-8.3]$ \\
\hline Bacteremia and sepsis (alone) & 202 & $15,563 \pm 18,856$ & 3837 [2184-5481] & $5.9[2.6-9.2]$ \\
\hline Other sites ${ }^{c}$ & 338 & $10,845 \pm 15,505$ & 2528 [1459-3596] & $4.6[2.1-7.1]$ \\
\hline
\end{tabular}

ESBL extended-spectrum $\beta$-lactamase, MRSA methicillin-resistant Staphylococcus aureus

${ }^{a}$ Other sites included: lower respiratory tract, gastrointestinal and abdominal, bone and joint, during pregnancy (mother), infection in newborn, heart and mediastinum, ear, nose and throat, eye, nervous system

${ }^{\mathrm{b}}$ Other sites included: skin and soft tissues, lower respiratory tract, bone and joint, during pregnancy (mother), infection in newborn, heart and mediastinum, ear, nose and throat, eye, nervous system

${ }^{c}$ Other sites included: skin and soft tissues, gastrointestinal and abdominal, bone and joint, during pregnancy (mother), infection in newborn, heart and mediastinum, ear, nose and throat, eye, nervous system 
EUR1424 per case for urinary and gynecological tract infection to EUR1688 for bacteremia. For infections involving ESBL pathogens, urinary and gynecological tract infections had the lowest differential cost, with a similar cost for $E$. coli and Klebsiella. However, the site of infection associated with the highest differential cost differed between these two pathogens. For ESBL E. coli, the highest differential cost was associated with gastrointestinal and abdominal infections, with a differential cost of EUR1439 per case, and an excess hospital stay of 2.3 days. The anatomical site associated with the highest differential cost for ESBL Klebsiella was lower respiratory tract infection (EUR5448 and 5.1 excess days in hospital). All results were significantly different from 0 , except for hospital days in excess induced by MRSA bacteremia with 1.3 extra days ( $95 \% \mathrm{CI}-0.2$ to 2.9 ).

Extrapolation of data from the matched-pair sample to the 46,839 stays associated with antibiotic-resistant infection and several coded infections led to an overall total excess cost of AMR for health insurance in 2015 at EUR109.3 million. Extrapolation of AMR to the 939,395 stays without identified pathogen, based on age, sex and infection site, led to a total cost of EUR177.8 million associated with 132,549 stays with AMR infection. By adding the total cost of AMR infection in our matched sample to the extrapolated costs, the overall excess hospital cost of AMR could reach EUR287.1 million in 2015. Thus, the excess cost of AMR could represent up to $3.2 \%$ of the total expenditure for hospital stays with infection, which amounted to EUR8.9 billion for 1648,566 stays in 2015.

Similar results were obtained using an unbalanced matching (1-5 controls per case) or a linear regression (Supplementary Appendix 4).

\section{Discussion}

The study determined the hospital cost of AMR from a payer perspective with the National French Hospital Discharge database PMSI. Our results suggest that the excess cost incurred by infection with AMR compared with a corresponding infection due to a susceptible microorganism, reached a mean differential cost of EUR1103 per stay. This result was extrapolated to all cases identified with AMR infection, and the excess expenditure associated with resistance amounted to EUR109.3 million. If all stays with AMR were identified in the PMSI, the overall cost could reach EUR287.1 million in 2015 or $3.2 \%$ of total expenditure for hospital stays with infection in France. We found that the estimated cost per case varied largely according to the anatomical site of infection and pathogens involved, but resistance remained consistently associated with excess costs, ranging from EUR691.1 for urinary and gynecological site to EUR2778.2 for primary bacteremia.
Our costs estimates are more modest than usually found in the literature. For example, the costs of infection with MRSA has been extensively investigated and total hospital costs in excess for infection with resistant strains as compared with suceptible strains were estimated at US\$2500 (EUR3201) [26] from a societal perspective or EUR8198 [21] from a payer perspective. Our cost estimates were an average of EUR1544. However, our results are very similar to the incremental cost for treatment of patients with AMR from the Medical Expenditure Panel Survey, which was estimated at US\$1383 (EUR1177) [27]. These differences could be explained in part by the difference between healthcare systems but also by the characteristics of our sample. Specifically, infections from urinary and gynecological sites were the most common sites in our study and caused the lowest differential cost. However, they might have been over-represented relative to other sites, with an unknown proportion of colonization rather than infection, possibly due to the common prescription of urine cultures leading to identification of AMR bacteria, whereas microbiological documentation at other sites is more difficult to obtain.

Nevertheless, our results appear to be consistent with trends from the literature. As in other studies, urinary tract infections were found to be the most frequent single infection [28], although to a lesser extent, and had the lowest increase in cost compared with other infections. The two most common infection sites for ESBL $E$. coli were urinary tract infections and gastrointestinal and abdominal infections in accordance with Melzer et al [29]. Finally, the in-hospital mortality (all-cause) rate was higher for patients with AMR in our study, in accordance with other studies $[29,30]$.

The present study had three main limitations. First, our data source depends on the coding quality of hospital records. Indeed, the main function of the PMSI is the management of hospital reimbursements. Therefore, trends in coding patterns may bias results in favor of hospitals for higher reimbursement per stays. Nevertheless, the French public insurance randomly checks the adequacy between coding records and the patient's actual files, which results in decreasing coding errors. ICD-10 codes referring to resistance can be recorded in the database for a given stay only if resistance is registered in the bacteriology records and if the resistance marker is expected to alter the management of the patient (i.e. extra antibiotic treatment, isolation precautions, etc.). Thus, we assume that the PMSI identifies the most severe cases with the greatest impact on care and on the national budget. However, it may not provide a full picture of antimicrobial resistance in hospitals. Second, these ICD10 codes for AMR were recently implemented and could be underused by hospitals, as shown in another study on administrative database [20]. An improvement of resistance coding is expected in coming years. Finally, since it was not possible within the PMSI database to link a resistance 
code and/or a pathogen to a given infection in cases with multiple infections or pathogens, such stays were excluded for our case-matched study and needed extrapolation to be secondarily included in the evaluation of the overall cost of resistance. This could lead to minimizing costs of AMR, in particular for the extrapolation by age and gender.

Matched case-control study is suitable in the "replacement scenario" [31] where AMR infections replace infections caused by susceptible microorganism, with no effect on the total burden. In the alternate scenario where infection due to AMR bacteria add to susceptible infections, costs of both burdens add up [32, 33], and estimation of the economic cost from the PMSI would require assessing costs attributable to AMR for stays where the main reason for hospitalization was not an infection. This would add uncertainty to the estimate and could lead to overestimate cost if antibiotic-resistant infections partially replace antibioticsusceptible infections, which may be untrue for MRSA [34]. Therefore, our estimate is a minimum.

This study was undertaken from the French public health insurance perspective, consequently our estimations may not reflect the actual costs to the hospital such as blocked beds or implementation of AMR control program at the expense of the hospital. In the context of antimicrobial studies, hospital perspective needs a micro-costing approach or robust empirical data and this perspective is needed to estimate a societal cost of AMR. But the estimated cost from the French public health insurance perspective provides an estimate of a minimum cost of AMR supported by the society. Indeed, the French public health insurance finances $86 \%$ of the expenses related to health and covers $92 \%$ of the French population. The hospital sector accounted for $41 \%$ of health expenditure for French public health insurance in 2015. For this reason, the payer's perspective is useful to policy makers despite the lack of data from the hospital perspective.

Nevertheless, our estimation has some strong advantages compared with existing studies. We used a national administrative database which avoids selection and information bias since this database covers the entire hospitalization in France. Second, we used a matched case-control approach controlling for age, sex, Charlson index, infections in principal diagnosis and hospital status to minimize confounding factors such as underlying comorbidities. We attempted to select cases and controls as similar as possible, where AMR presence was the only differential factor. As demonstrated with the linear regression model, the low number of unmatched stays $(<2 \%)$ limited the magnitude of the impact on our results of patients excluded from the matched-pair analysis, despite their highest cost of stays and more severe health status.

We have shown that it is possible to select AMR cases within a large medico-administrative database. The methods used in this study could be routinely replicated as an economic indicator of AMR. Thus, our results could be used for international comparison studies, although the average cost per case was calculated from a French cost index. It may be relevant to compare the investments of different insurance systems in the management of AMR in hospitals across different countries. Finally, in the absence of specific data on hospital costs for a wide range of infections, our study could be used in economic burden [5] or future projection [35] since the number of additional hospital days induced by AMR should be comparable in others European countries.

In summary, our work reveals the amount of public health system spending devoted to care of patients with antibiotic-resistant infections in a developed country. Our study confirms the need to reinforce and expand programs for prevention of AMR infection. Future studies based on empirical data are needed to evaluate the loss of productivity and the fate of patients after discharge from the hospital to encompass the societal perspective and have a complete understanding of AMR cost.

Acknowledgements We are grateful to the Maitrise d'Ouvrage Informatique Stratégie et Etudes (MOISE/DEMEX) Department of the French National Health Insurance for providing the database.

Author Contributions LW, DG, and CB-B designed the study. KC, JS, GdL, MT, LW, PT and MO established the code list. JS, CB-B, $\mathrm{KC}, \mathrm{LW}$ and $\mathrm{MO}$ defined the selection and recoding rules. MO and MT developed the algorithm. MT, LW, CB-B, GdL and PT analysed the data. MT wrote the first draft of the manuscript. MT, LW, CB-B, GdL and PT drafted the manuscript. All authors read, amended and approved the final manuscript.

\section{Compliance with Ethical Standards}

Financial support This work was supported by the French Ministry of Health.

Conflict of interest The funders were not involved in the study design, data collection or analysis. The decision to publish and the preparation of the manuscript were only the authors' own will. All authors (M, Touat, M Opatowski, C. Brun-Buisson, K. Cosker, D. Guillemot, J. Salomon, P. Tuppin, G de Lagasnerie and L. Watier) report no conflicts of interest relevant to this article.

Data availability statements Data available on request from the authors

Open Access This article is distributed under the terms of the Creative Commons Attribution-NonCommercial 4.0 International License (http://creativecommons.org/licenses/by-nc/4.0/), which permits any noncommercial use, distribution, and reproduction in any medium, provided you give appropriate credit to the original author(s) and the source, provide a link to the Creative Commons license, and indicate if changes were made. 


\section{References}

1. Laxminarayan R, Duse A, Wattal C, et al. Antibiotic resistancethe need for global solutions. Lancet Infect Dis. 2013;13:1057-98.

2. World Health Organization. WHO global strategy for containment of antimicrobial resistance. WHO/CDS/CSR/DRS/2001. 2. Geneva: The Organization; 2001. http://www.who.int/csr/resou rces/publications/drugresist/EGlobal_Strat.pdf.

3. Carmeli Y, Eliopoulos G, Mozaffari E, Samore M. Health and economic outcomes of vancomycin-resistant enterococci. Arch Intern Med. 2002;162:2223-8.

4. Cosgrove SE, Qi Y, Kaye KS, et al. The impact of methicillin resistance in Staphylococcus aureus bacteremia on patient outcomes: mortality, length of stay, and hospital charges. Infect Control Hosp Epidemiol. 2005;26:166-74.

5. Europäisches Zentrum für die Prävention und die Kontrolle von Krankheiten, editor. The bacterial challenge, time to react: a call to narrow the gap between multidrug-resistant bacteria in the EU and the development of new antibacterial agents. Stockholm: ECDC; 2009.

6. Colomb-Cotinat M, Lacoste J, Brun-Buisson C, et al. Estimating the morbidity and mortality associated with infections due to multidrug-resistant bacteria (MDRB), France, 2012. Antimicrob Resist Infect Control. 2016;5:56.

7. de Kraker MEA, Jarlier V, Monen JCM, et al. The changing epidemiology of bacteraemias in Europe: trends from the European Antimicrobial Resistance Surveillance System. Clin Microbiol Infect. 2013;19:860-8.

8. Brolund A, Edquist PJ, Mäkitalo B, et al. Epidemiology of extended-spectrum $\beta$-lactamase-producing Escherichia coli in Sweden 2007-2011. Clin Microbiol Infect. 2014;20:O344-52.

9. Edelsberg J, Taneja C, Zervos M, et al. Trends in US hospital admissions for skin and soft tissue infections. Emerg Infect Dis. 2009;15:1516-8.

10. Diekema DJ, BootsMiller BJ, Vaughn TE, et al. Antimicrobial resistance trends and outbreak frequency in United States hospitals. Clin Infect Dis. 2004;38:78-85.

11. Jo C. Cost-of-illness studies: concepts, scopes, and methods. Clin Mol Hepatol. 2014;20:327-37.

12. Smith R, Coast J. The true cost of antimicrobial resistance. BMJ. 2013;346:f1493.

13. Roberts RR, Hota B, Ahmad I, et al. Hospital and societal costs of antimicrobial-resistant infections in a Chicago teaching hospital: implications for antibiotic stewardship. Clin Infect Dis. 2009;49:1175-84.

14. Kopp BJ, Nix DE, Armstrong EP. Clinical and economic analysis of methicillin-susceptible and -resistant Staphylococcus aureus infections. Ann Pharmacother. 2004;38:1377-82.

15. Park SY, Son JS, Oh IH, Choi JM, Lee MS. Clinical impact of methicillin-resistant Staphylococcus aureus bacteremia based on propensity scores. Infection. 2011;39:141.

16. Webb M, Riley LW, Roberts RB. Cost of hospitalization for and risk factors associated with vancomycin-resistant Enterococcus faecium infection and colonization. Clin Infect Dis. 2001;33:445-52.

17. Cosgrove SE. The relationship between antimicrobial resistance and patient outcomes: mortality, length of hospital stay, and health care costs. Clin Infect Dis. 2006;42(Suppl 2):S82-9.

18. Naylor NR, Atun R, Zhu N, et al. Estimating the burden of antimicrobial resistance: a systematic literature review. Antimicrob Resist Infect Control. 2018;7:58. https://doi.org/10.1186/s1375 6-018-0336-y.

19. Andreassen AES, Jacobsen CM, de Blasio B, et al. The impact of methicillin-resistant $S$. aureus on length of stay, readmissions and costs: a register based case-control study of patients hospitalized in Norway. Antimicrob Resist. Infect Control. 2017;6:1-74.

20. Macedo-Viñas M, De Angelis G, Rohner P, et al. Burden of meticillin-resistant Staphylococcus aureus infections at a Swiss University hospital: excess length of stay and costs. J Hosp Infect. 2013;84:132-7.

21. Resch A, Wilke M, Fink C. The cost of resistance: incremental cost of methicillin-resistant Staphylococcus aureus (MRSA) in German hospitals. Eur J Health Econ. 2009;10:287-97.

22. Scheller-Kreinsen D, Quentin W, Busse R. DRG-based hospital payment systems and technological innovation in 12 European countries. Value Health. 2011;14:1166-72.

23. Abramson M, Sexton D. Nosocomial methicillin-resistant and methicillin-susceptible Staphylococcus aureus primary bacteremia: at what costs? Infect Control Hosp Epidemiol. 1999;20(6):408-11.

24. Chaix C, Durand-Zaleski I, Alberti C, Brun-Buisson C. Control of endemic methicillin-resistant Staphylococcus aureus: a cost-benefit analysis in an intensive care unit. JAMA. 1999;282:1745-51.

25. Tuppin P, Rudant J, Constantinou P, et al. Value of a national administrative database to guide public decisions: From the système national d'information interrégimes de l'Assurance Maladie (SNIIRAM) to the système national des données de santé (SNDS) in France. Rev Epidemiol Sante Publique. 2017;65(Suppl 4):S149-67.

26. Rubin RJ, Harrington CA, Poon A, et al. The economic impact of Staphylococcus aureus infection in New York City hospitals. Emerg Infect Dis. 1999;5:9-17.

27. Thorpe KE, Joski P, Johnston KJ. Antibiotic-resistant infection treatment costs have doubled since 2002, now exceeding $\$ 2$ billion annually. Health Aff Proj Hope. 2018;37:662-9.

28. Plowman R, Graves N, Griffin MA, et al. The rate and cost of hospital-acquired infections occurring in patients admitted to selected specialties of a district general hospital in England and the national burden imposed. J Hosp Infect. 2001;47:198-209.

29. Melzer M, Petersen I. Mortality following bacteraemic infection caused by extended spectrum beta-lactamase (ESBL) producing E. coli compared to non-ESBL producing E. coli. J Infect. 2007;55:254-9.

30. Cosgrove SE, Sakoulas G, Perencevich EN, et al. Comparison of mortality associated with methicillin-resistant and methicillinsusceptible Staphylococcus aureus bacteremia: a meta-analysis. Clin Infect Dis. 2003;36:53-9.

31. Linnemann CC, Moore P, Staneck JL, Pfaller MA. Reemergence of epidemic methicillin-resistant Staphylococcus aureus in a general hospital associated with changing Staphylococcal strains. Am J Med. 1991;91:S238-44.

32. Mostofsky E, Lipsitch M, Regev-Yochay G. Is methicillin-resistant Staphylococcus aureus replacing methicillin-susceptible $S$. aureus? J Antimicrob Chemother. 2011;66:2199-214.

33. Ammerlaan HSM, Harbarth S, Buiting AGM, Crook DW, Fitzpatrick $\mathrm{F}$, Hanberger $\mathrm{H}$, et al. Secular trends in nosocomial bloodstream infections: antibiotic-resistant bacteria increase the total burden of infection. Clin Infect Dis. 2013;56:798-805.

34. Boyce JM, White RL, Spruill EY. Impact of methicillin-resistant Staphylococcus aureus on the incidence of nosocomial staphylococcal infections. J Infect Dis. 1983;148:763.

35. Taylor J, Hafner M, Yerushalmi E, et al. Estimating the economic costs of antimicrobial resistance: model and results, The Wellcome Trust, RR-911-WT; 2014. https://www.rand.org/pubs/resea rch_reports/RR911.html. Accessed 17 May 2017. 